\title{
Microbiological Profile and Drug Sensitivity Pattern in Persistently Discharging Ears: A Tertiary Level Hospital Based Retrospective Study
}

\author{
Bigyan R Gyawali', Rabindra B Pradhanaga', Pabina Rayamjhi', Kripa Dongol', Mudit Gupta1, \\ Lava Shrestha ${ }^{2}$, Kunjan Acharya ${ }^{1}$ \\ 'Department of ENT, Head and Neck Surgery, Maharajgunj Medical Campus, Ganesh Man Singh Memorial \\ Academy of ENT, Head \& Neck Studies, Tribhuvan University Teaching Hospital, Institute of Medicine, Maharajgunj, \\ Kathmandu, Nepal, ${ }^{2}$ Department of Clinical Physiology, Maharajgunj Medical Campus, Institute of Medicine, \\ Maharajgunj, Kathmandu, Nepal.
}

\section{Corresponding author:}

\section{Bigyan R Gyawali, MS, MRCS}

Department of ENT, Head and Neck Surgery, Maharajgunj Medical Campus, Ganesh Man Singh Memorial Academy of ENT, Head \& Neck Studies, Tribhuvan University Teaching Hospital, Institute of Medicine, Maharajgunj, Kathmandu, Nepal

Email: bigyan.gyawali@gmail.com

Submitted : Jun 14, 2020

Accepted : Jul 28, 2020

\begin{abstract}
\section{Introduction}

Antimicrobial resistance is an emerging issue resulting from the misuse of antibiotics in today's world. As an effect of it, cases with persistent ear discharge not responding to the conventional oral antibiotics prescribed in ear pathologies are seen increasing in our day to-day practice. This study aims to review the microbiological growth and the pattern of their antibiotic sensitivity in this group of population.

\section{Methods}

This was a retrospective study which was conducted at the Department of ENT-HNS of Tribhuvan University Teaching Hospital. Record files of all cases who presented with persistently discharging ear for more than 2 weeks and not responding to oral antibiotics, between January 2017 to March 2020 were assessed. Cases of chronic otitis media (COM) squamous with persistent discharge were excluded as most of the times it is the disease process rather than type of organism which causes the persistence of ear discharge despite the use of antibiotics.
\end{abstract}

\section{Results}

COM mucosal and cavity infection following modified radical mastoidectomy (MRM) were the commonest pathologies. Pseudomonas aeruginosa (PA) and Coagulase negative Staphylococcus (CoNS) were the commonest bacterial isolates. Pseudomonas aeruginosa showed a good sensitivity to antibiotics like Tazobactam and Piperacillin and resistance to conventionally used antibiotics like Ciprofloxacin and Levofloxacin. Similarly, CoNS had good sensitivity to antibiotics like Oxacillin and Levofloxacin and resistance to conventionally used antibiotics like Amoxicillin.

\section{Conclusion}

COM mucosal and post MRM cavity infection are the commonest pathologies presenting with persistent ear discharge with PA and CoNS as the predominant isolates. Pseudomonas aeruginosa holds a good sensitivity to drugs like Tazobactam and Piperacillin; and CoNS to drugs like Oxacillin and Levofloxacin.

Keywords: Drug sensitivity, ear discharge, microbiological, persistent 


\section{INTRODUCTION}

E ar discharge is one of the common otological presentations we face in our daily practice. Moreover, untreated ear infection is one of the leading causes of hearing loss in the developing countries. ${ }^{1}$ Acute otitis media (AOM), otitis externa $(\mathrm{OE})$, myringitis, chronic otitis media (COM) mucosal and COM squamous are the common causes of ear discharge. While pathologies like AOM, OE and myringitis are managed solely with antibiotics, COM on the other hand needs both medical and surgical management. The common known bacterial isolates in these pathologies are Pseudomonas aeruginosa (PA), Escherichia coli, Staphylococcus aureus (SA), Streptococcus pyogenes (SP), Proteus mirabilis (PM), Klebsiella spp., or mixed bacterial infection. ${ }^{2}$

An easy accessibility of antibiotics as an over the counter drug and thus their misuse and also a lack of standardized antibiotic protocols in our part of the world has led to an emergence of various antibiotic resistant organisms. As an effect of this, cases with persistent ear discharge not responding to conventionally prescribed oral antibiotics for ear pathologies are increasing.

The objective of this study was to review the microbiological growth and the pattern of their antibiotic sensitivity in the cases presenting with persistently discharging ear, not responding with oral antibiotics.

\section{METHODS}

This was a retrospective study which was conducted at the Department of ENT-HNS of Maharajgunj Medical Campus, Tribhuvan University Teaching Hospital. The study was approved by the Institutional Review Committee of Institute of Medicine. Data of demographic profile, nature of ear pathology and report of pus culture and sensitivity (C/S) of all cases who present with persistently discharging ear for more than 2 weeks and not responding to oral antibiotics are recorded at our department. These prospectively set data between January 2017 to March 2020 were assessed.

All such cases were advised with oral fluoroquinolones if they had COM mucosal or myringitis and Amoxicillin if they had AOM for 10 days along with topical antibiotic ear drops (a combination of Chloramphenicol and Polymixin $B$ with Dexamethasone or combination of Betamethasone with Neomycin) as per the departmental protocol. If no response was seen, antibiotics were stopped for 72 hours and then pus C/S was sent.

Pus from external auditory and middle ear was collected with the sterile technique. The culture was done in Blood Agar and MacConkey's Agar with incubation at $37^{\circ} \mathrm{C}$ for 72 hours. Antibiotic sensitivity was performed in Mueller Hinton Agar. We used The Clinical \& Laboratory Standards Institute (CLSI) Guidelines to perform the antibiotic sensitivity.

\section{RESULTS}

A total of 55 cases were included in the study. M: F was 2:1 and the majority of the cases were below 30 years (Table 1 ).

Table 1. Age distribution of cases

\begin{tabular}{cc}
\hline Age group & Number \\
\hline$<30$ years & 28 \\
$30-60$ years & 25 \\
$>60$ years & 2 \\
\hline
\end{tabular}

COM mucosal was the commonest pathology followed by post-MRM cavity infection. Similarly, PA was the commonest organism to be isolated followed by CoNS. While CoNS could be cultured in all pathologies i.e., AOM, Chronic myringitis, COM mucosal and post-MRM cavity infection, PA growth was predominantly seen in cases with COM mucosal and post-MRM cavity infection (Table 2).

Table 2. Bacterial growth in pus $\mathrm{C} / \mathrm{S}$ in different ear pathologies

\begin{tabular}{lcccc}
\hline & \multicolumn{4}{c}{ No. of growth in } \\
\cline { 2 - 5 } $\begin{array}{c}\text { Bacterial } \\
\text { organism }\end{array}$ & AOM & $\begin{array}{c}\text { Chronic } \\
\text { myringitis }\end{array}$ & $\begin{array}{c}\text { COM } \\
\text { mucosal }\end{array}$ & $\begin{array}{c}\text { Post-MRM } \\
\text { with cavity } \\
\text { infection }\end{array}$ \\
\hline CoNS & & & & 7 \\
P. aeruginosa & - & - & 30 & 1 \\
S. maltophilia & - & - & - & 8 \\
Enterobacter & - & - & 1 & 1 \\
MRSA & - & - & 1 & - \\
S. pyogenes & - & - & 1 & - \\
No growth & - & - & 2 & - \\
\hline
\end{tabular}

CoNS: Coagulase negative Staphylococcus; Pseudomonas aeruginosa; Stenotrophomonas maltophilia; Methicillin resistant Staphylococcus aureus; Streptococcus pyogenes

An incomplete documentation and also a nonuniform use of different antibiotic discs for culture sensitivity was a major cause for an uneven distribution of the numbers related to the sensitivity of the bacteria to different antibiotics as seen in Table 3. As PA and CoNS were the major isolates, analysis of the drug sensitivity pattern was confined to these two organisms.

Considering the number of tests done, PA showed a good sensitivity to drugs like Tazobactam (100\%), 
Table 3. Sensitivity pattern of Pseudomonas aeruginosa and Coagulase negative Staphylococcus to different drugs

\begin{tabular}{|c|c|c|c|c|}
\hline \multirow{3}{*}{$\begin{array}{l}\text { Bacterial } \\
\text { organism }\end{array}$} & \multicolumn{4}{|c|}{ Drug sensitivity of bacteria } \\
\hline & \multicolumn{2}{|c|}{ Pseudomonas aeruginosa } & \multicolumn{2}{|c|}{ Coagulase -ve Staphylococcus } \\
\hline & Sensitive & Resistant & Sensitive & Resistant \\
\hline Amoxicillin & - & - & - & $6(100 \%)$ \\
\hline Ciprofloxacin & $4(12.5 \%)$ & $28(87.5 \%)$ & $6(60 \%)$ & $4(40 \%)$ \\
\hline Levofloxacin & $7(21.8 \%)$ & $25(78.2 \%)$ & $5(83.3 \%)$ & $1(16.7 \%)$ \\
\hline Doxycycline & $1(50 \%)$ & $1(50 \%)$ & $2(66.6 \%)$ & $1(33.3 \%)$ \\
\hline Cephalexim & $1(50 \%)$ & $1(50 \%)$ & $9(81.8 \%)$ & $2(18.2 \%)$ \\
\hline Cefixime & $2(50 \%)$ & $2(50 \%)$ & $1(100 \%)$ & - \\
\hline Ceftriaxone & - & $1(100 \%)$ & - & $1(100 \%)$ \\
\hline Ceftazidime & $24(75 \%)$ & $8(25 \%)$ & - & - \\
\hline Cefoperazone & $4(80 \%)$ & $1(20 \%)$ & - & - \\
\hline Cloxacillin & - & - & $9(90 \%)$ & $1(10 \%)$ \\
\hline Amikacin & $18(51.4 \%)$ & $17(48.5 \%)$ & $3(37.5 \%)$ & $5(62.5)$ \\
\hline Gentamicin & $8(27.5 \%)$ & $21(72.4 \%)$ & $5(55.5 \%)$ & $4(44.4 \%)$ \\
\hline Tigicycline & - & - & $1(50 \%)$ & $1(50 \%)$ \\
\hline Meropenem & $7(87.5 \%)$ & $1(12.5 \%)$ & - & - \\
\hline Imipenem & $7(100 \%)$ & 0 & - & - \\
\hline Chloramphenicol & 0 & $2(100 \%)$ & $2(66.6 \%)$ & $1(33.3 \%)$ \\
\hline Teicoplanin & - & - & $1(50 \%)$ & $1(50 \%)$ \\
\hline Clindamycin & - & - & $5(62.5 \%)$ & $3(37.5 \%)$ \\
\hline Erythromycin & - & - & $1(14.2 \%)$ & $7(87.5 \%)$ \\
\hline Cotrimoxazole & - & $1(100 \%)$ & 2 (33.3\%) & $4(66.6 \%)$ \\
\hline Tazobactam & $38(100 \%)$ & 0 & $2(66.6)$ & $1(33.3 \%)$ \\
\hline Piperacillin & $38(100 \%)$ & 0 & $1(33.3 \%)$ & $2(66.6 \%)$ \\
\hline Polymixin & $10(100 \%)$ & 0 & $1(50 \%)$ & $1(50 \%)$ \\
\hline Colistin & $10(100 \%)$ & 0 & - & 1 \\
\hline
\end{tabular}

Piperacillin (100\%), Polymixin (100\%), Colistin (100\%), Imipenem (100\%), Meropenem (87.5\%), Cefoperazone $(80 \%)$ and Ceftazidime $(75 \%)$. There was a relatively high resistance to commonly used anti-pseudomonal drugs like Ciprofloxacin (87.5\%), Levofloxacin (78.2\%), and Gentamicin (72.4\%). Similarly, CoNS showed good sensitivity towards antibiotics like Cloxacillin (90\%), Cephalexin (81.8\%), Levofloxacin (83.3\%), Clindamycin (62.5\%) and Ciprofloxacin (60\%). Significant resistance was seen in antibiotics like Amoxicillin (100\%) and Erythromycin (87.5\%). A complete antibiotic sensitivity pattern of these bacterial isolates is shown in Table 3.

\section{DISCUSSION}

This study was focused on the pattern of bacterial growth and their sensitivity to various antibiotics in cases with persistently discharging ears not responding to conventional oral antibiotics.

The commonest pathology prevalent amongst our study population was COM mucosal and the commonest bacterial isolate was PA i.e. in $69 \%$ of the cases. CoNS was the next common isolate seen in $20 \%$ of cases. The other bacteria namely; Enterobacter, Methicillin Resistant Staphylococcus
Aureus (MRSA) and Streptococcus were isolated in one case each. Two cases didn't have any growth in culture. PA had a very good sensitivity to drugs like Tazobactam, Piperacillin, Polymixin, Colistin, Imipenem, Meropenem, Cefoperazone and Ceftazidime. However, ithadaverypoorsusceptibility to fluoroquinolones and aminoglycosides. On the other hand, CoNS had good sensitivity to Cloxacillin and fluoroquinolones with resistance to Amoxicillin and Erythromycin. A study from India by Vishwanath et al. showed PA, SA and CoNS as the commonest organisms to be isolated in cases with COM. Pseudomonas Aeruginosa had a good sensitivity to drugs like Ceftazidime, Piperacillin, Ciprofloxacin, Trobramycin, Amikacin and Gentamicin. However, it showed a very poor susceptibility to Neomycin. Similar was the case with CoNS, which showed a good sensitivity to all antibiotics except for Neomycin. ${ }^{3}$ Similarly, in a study from Saudi Arabia, amongst cases with COM, Methicillin Sensitive Staphylococcus Aureus (MSSA) was the commonest isolate followed by PA, MRSA, CoNS, PM and E. Coli. The gram positive organisms in their study showed a good sensitivity to antibiotics like fluoroquinolones, aminoglycosides and Bacitracin and resistance to antibiotics like Penicillin and Ampicillin. Pseudomans Aeruginosa, similar to our 
study, showed a very good sensitivity to drugs like Ceftazidime and Piperacillin. Also, they had a good sensitivity to fluoroquinolones and aminoglycosides which, however, was a contradictory finding to our study. ${ }^{4}$ One of the reasons for the discrepancy seen in the susceptibility pattern of the organisms between these studies and ours could be the selective inclusion of the cases not responding to oral antibiotics in our study.

Cavity infection following MRM can be cumbersome to the patient most of the times. Various factors play a role in the persistence of discharge in these cases. A large mastoid cavity, high facial ridge, inadequate meatoplasty, residual or recurrent disease are the note worthy ones. Apart from anatomical factors, drug resistant microbial growth is another factor. ${ }^{5,6}$ In our study, we excluded cases with the obvious pathology which could render persistence of discharge. Of the ten cases with cavity infection, eight had PA isolated in the culture. Coagulase -ve Staphylococcus and Stenotrophomonas Maltophilia were isolated in one case each. In a study by Dutta et al. in pediatric cases with persistent cavity infection following MRM, 22 of 50 cases showed a positive bacterial growth while rest 28 had a sterile culture. Of those cases with positive bacterial isolation, PA was the most common i.e. in 14 cases. Also, in accordance to our study, it showed a good sensitivity to Piperacillin and Ceftazidime. ${ }^{7}$ In contrast to our study, a comparative study by An-ting et al. between pre and post-operative microbiological profile in cases undergoing revision mastoid surgery, CoNS was the most common isolate in both groups. ${ }^{8}$

Chronic myringitis (CM) refers to chronic inflammation of the tympanic membrane with or without involvement of bony external auditory canal. ${ }^{9}$ The clinical presentation often masquerades COM squamous, however, most of the times it resolves with topical and oral antibiotics. Various studies have shown the commonest isolates to be SA and PA. ${ }^{10,11}$ In our study, there were two cases of $\mathrm{CM}$ with persistent ear discharge and in both of them the culture yielded CoNS.

AOM with persistent ear discharge is relatively rare as most of the times it responds well with oral antibiotics. Around $60 \%$ of the cases have identifiable bacterial cause. ${ }^{12}$ In our study, only one case with persistent ear discharge was found to have AOM with CoNS as an isolate. In a study by Smith et al., of 38 cases with AOM with ear discharge, only 22 cases had bacteria isolated on culture with SA followed by Streptococcus being the predominant ones. Hemophilus Influenzae and PA were isolated in very few cases..$^{12}$ An African study by Gorems et al. showed SA, Citrobacter and CoNS as the predominant bacterial isolates in cases with AOM. The gram positive isolates in their study had a good sensitivity to Ceftazidime, Ciprofloxacin and Gentamicin and an increased level of resistance to Co-trimoxazole, Aminopenicillins and Penicillin-G. ${ }^{13}$

Pseudomonas aeruginosa, a gram negative rod, is an ubiquitous organism capable of surviving wide range of environmental conditions. ${ }^{14}$ The emerging resistance of this organism to various antibiotics is posing a great challenge at today's scenario. WHO has even warranted a need of new antibiotics to treat Carbepenem resistant PA. ${ }^{15}$ Various mechanisms are known to cause the drug resistance. Intrinsic mechanisms like poor outer membrane permeability to the drugs, inactivation of the drugs by intrinsic enzymes and efflux of the drug by pumping mechanisms, acquired resistance by mutational changes and genetic transfer and also by the formation of biofilms rendering an impermeable barrier to the antibiotics are the known ones. ${ }^{16,17}$ Coagulase -ve Staphylococcus is the normal commensal of skin and mucus membrane. And their isolation, most of the times, signifies rather a possibility of contamination. However, these commensals are often known to cause infection in immune compromised hosts and neonates. ${ }^{18}$ In our study it is difficult to rule out the possibility of contamination, however, considering a good response of all the cases with CoNS to C/S guided antibiotics and the evidence of isolation of CoNS in the above discussed studies from different geographical regions suggest it can have a pathogenic role in middle ear infections. 3,4,8,13

This study had various limitations. As it was a retrospective study, incomplete documentation was one of the flaws. Also, anaerobic growth wasn't assessed in all cases due to unavailability of the necessary equipments at our center.

\section{CONCLUSION}

Cases with persistent ear discharge not responding to commonly used oral antibiotics i.e. fluoroquinolones and Amoxicillin are increasing owing to emerging antimicrobial resistance. COM mucosal and post MRM cavity infection are the commonest pathologies presenting with persistent ear discharge with PA and CoNS as the predominant isolates. Pseudomonas aeruginosa holds a good sensitivity to drugs like Tazobactam and Piperacillin and CoNS to drugs like Oxacillin and Levofloxacin.

\section{CONFLICT OF INTEREST}

None declared.

\section{REFERENCES}

1. Argaw-Denboba A, Abejew AA, Mekonnen AG. AntibioticResistant Bacteria Are Major Threats of Otitis Media in Wollo Area, Northeastern Ethiopia: A Ten-Year Retrospective Analysis. Int J Microbiol. 2016;2016. doi:10.1155/2016/8724671 
2. Afolabi O, Salaudeen A, Ologe F, Nwabuisi C, Nwawolo C. Pattern of bacterial isolates in the middle ear discharge of patients with chronic suppurative otitis media in a tertiary hospital in North central Nigeria. Afr Health Sci. 2012;12(3):362-367. Accessed July 3, 2020. https://www.ncbi.nlm.nih.gov/pmc/articles/PMC3557674/

3. Vishwanath S, Mukhopadhyay C, Prakash R, Pillai S, Pujary K, Pujary P. Chronic suppurative otitis media: optimizing initial antibiotic therapy in a tertiary care setup. Indian J Otolaryngol Head Neck Surg. 2012;64(3):285-289. doi:10.1007/s12070-011-0287-6

4. Ahmad S. Antibiotics in chronic suppurative otitis media: A bacteriologic study. Egyptian Journal of Ear, Nose, Throat and Allied Sciences. 2013;14(3):191-194. doi:10.1016/j.ejenta.2013.06.001

5. East $C A$, Brough MD, Grant HR. Mastoid obliteration with the temporoparietal fascia flap. J Laryngol Otol. 1991;105(6):417-420. doi:10.1017/s0022215100116184

6. Gopalakrishnan S, Chadha SK, Gopalan G, Ravi D. Role of mastoid obliteration in patients with persistent cavity problems following modified radical mastoidectomy. J Laryngol Otol. 2001;115(12):967-972. doi:10.1258/0022215011909783

7. Dutta HD, Rayamajhi P, Dutta D. Modified radical mastoidectomy in children: mastoid cavity problem and its management. International Journal of Otorhinolaryngology and Head and Neck Surgery. 2019;5(5):1156-1160. doi:10.18203/issn.2454-5929. ijohns20193157

8. An-ting X, Ming X, Han-bing Z, Kimitaga K. Bacteriolgy before and after total middle ear reconstruction. Journal of Otology. 2007;2(2):114-118. doi:10.1016/S1672-2930(07)50023-1

9. Devaraja K. Myringitis: An update. J Otol. 2019;14(1):26-29. doi:10.1016/j.joto.2018.11.003

10. LeviJR,AmesJA, Gitman L, MorletT,O'ReillyRC. Clinical characteristics of pediatric granular myringitis. Otolaryngol Head Neck Surg.
2013;148(2):291-296. doi:10.1177/0194599812470433

11. Kim YH. Clinical characteristics of granular myringitis treated with castellani solution. Eur Arch Otorhinolaryngol. 2011;268(8):11391146. doi:10.1007/s00405-011-1500-7

12. Smith L, Ewings P, Smith C, Thompson M, Harnden A, Mant D. Ear discharge in children presenting with acute otitis media: observational study from UK general practice. Br J Gen Pract. 2010;60(571):101-105. doi:10.3399/bjgp10X483148

13. Gorems K, Beyene G, Berhane M, Mekonnen Z. Antimicrobial susceptibility patterns of bacteria isolated from patients with ear discharge in Jimma Town, Southwest, Ethiopia. BMC Ear Nose Throat Disord. 2018;18. doi:10.1186/s12901-018-0065-0

14. Silby MW, Winstanley C, Godfrey SAC, Levy SB, Jackson RW. Pseudomonas genomes: diverse and adaptable. FEMS Microbiol Rev. 2011;35(4):652-680. doi:10.1111/j.15746976.2011.00269.x

15. Pang Z, Raudonis R, Glick BR, Lin T-J, Cheng Z. Antibiotic resistance in Pseudomonas aeruginosa: mechanisms and alternative therapeutic strategies. Biotechnology Advances. 2019;37(1):177192. doi:10.1016/j.biotechadv.2018.11.013

16. Breidenstein EBM, de la Fuente-Núñez C, Hancock REW. Pseudomonas aeruginosa: all roads lead to resistance. Trends in Microbiology. 2011;19(8):419-426. doi:10.1016/j. tim.2011.04.005

17. Drenkard E. Antimicrobial resistance of Pseudomonas aeruginosa biofilms. Microbes and Infection. 2003;5(13):1213-1219. doi:10.1016/j.micinf.2003.08.009

18. May L, Klein EY, Rothman RE, Laxminarayan R. Trends in Antibiotic Resistance in Coagulase-Negative Staphylococci in the United States, 1999 to 2012. Antimicrobial Agents and Chemotherapy. 2014;58(3):1404-1409. doi:10.1128/AAC.01908-13 